\title{
Mittels aerosolbasierter Kaltabscheidung bei Raumtemperatur hergestellte schichtförmige NTC-Thermistorbauelemente
}

\author{
Michaela Schubert ${ }^{1}$, Christian Reichl ${ }^{1}$, Christian Münch ${ }^{2}$, Jaroslaw Kita ${ }^{1}$, Ralf Moos ${ }^{1}$ \\ ${ }^{1}$ Lehrstuhl für Funktionsmaterialien, Universität Bayreuth, 95440 Bayreuth, Germany \\ ${ }^{2}$ Vishay Electronic GmbH, Dr.-Felix-Zandman-Platz 1, 95100 Selb, Germany \\ funktionsmaterialien@uni-bayreuth.de; Tel.: 49 (0)921 557401
}

\section{Zusammenfassung}

In der vorliegenden Arbeit konnte gezeigt werden, dass eine aerosolbasierte Kaltabscheidung eines produktionsnahen NTCR-Keramikpulvers zu mechanisch stabilen Schichten möglich ist. Die Schichten zeichnen sich durch eine dichte nanokristalline Schichtstruktur und eine sehr gute Substratanbindung aus. Im ersten Versuchsteil konnte sowohl auf Edelstahl- als auch auf $\mathrm{Al}_{2} \mathrm{O}_{3}$-Substraten ein linearer Zusammenhang zwischen der erzielten Schichtdicke der aerosolkaltabgeschiedenen NTCR-Schicht und der Anzahl an Überfahrtsschleifen nachgewiesen werden. Basierend auf diesen Erkenntnissen wurden im zweiten Versuchsteil chipbasierte NTCR-Bauteile der Baugröße 1206 mit 10 und 20 Überfahrtsschleifen hergestellt. Die aerosolbasierte Kaltabscheidung des produktionsnahen Keramikpulvers erfolgte hierzu auf ein mit Sollbruchkanten und siebgedruckten Au-Interdigitalelektrodenstrukturen versehenes $\mathrm{Al}_{2} \mathrm{O}_{3}$-Substrat. Die Bauteile zeigen reproduzierbar die typische Charakteristik von konventionell gesinterten NTCR-Volumenkeramiken. Auch konnte der Nachweis erbracht werden, dass durch die Variation der Anzahl an Überfahrtsschleifen die Schichtdicke der aerosolkaltabgeschiedenen NTCR-Schicht und damit deren elektrische Widerstand gezielt eingestellt werden kann. Die charakteristische $B$-Konstante ändert sich dabei nicht.

Keywords: Aerosol Deposition Method (ADM), NTC Thermistor, Temperatursensor, Dickschicht

\section{Einleitung}

Bei NTC-Thermistoren (Negative Temperature Coefficient Thermally Sensitive Resistor [1]) handelt es sich größtenteils um halbleitende Keramiken auf Basis von Übergangsmetalloxiden, meist Übergangsmetallmanganaten mit Spinellstruktur $\mathrm{MeMn}_{2} \mathrm{O}_{4}(\mathrm{Me}=\mathrm{Ni}, \mathrm{Co}, \mathrm{Fe}, \mathrm{Cu}$, etc.) [2]. Diese zeichnen sich durch einen hohen negativen Temperaturkoeffizienten, geringe Kosten und eine hohe Zuverlässigkeit aus $[3,4]$. Angewendet werden NTC-Thermistoren vor allem zur präzisen Temperaturmessung und -kontrolle. Die große Temperaturabhängigkeit des elektrischen Widerstandes $R$ der spinellbasierten NTC-Thermistoren basiert auf einem phononengestützten Hüpfmechanismus von Ladungsträgern entlang lokalisierter Zustände, dem sog. Small Polaronen Hopping [5]. Das $R$ - $T$-Verhalten kann vereinfacht über eine Arrhenius-Beziehung (GI. (1)) beschrieben werden.

$$
R(T)=R_{0} \cdot \exp \left(E_{\mathrm{A}} /\left(k_{\mathrm{B}} \cdot T\right)\right)
$$

$R_{0}$ stellt dabei den elektrischen Widerstand bei unendlich hoher Temperatur, $E_{\mathrm{A}}$ die Aktivierungsenergie für den Hüpfprozess, $k_{B}$ die Boltzmann-Konstante und $T$ die Temperatur dar $[6,7]$. Aus dem Verhältnis $E_{A} / k_{B}$ ergibt sich die Thermistorkonstante $B$. Die $B$-Konstante und der elektrische Widerstand bei $25^{\circ} \mathrm{C}$ sind charakteristische Kenngrößen für die Beschreibung und den Vergleich von NTCThermistoren. Derzeit erfolgt die Herstellung der keramischen NTC-Thermistoren über traditionelle, sinterbasierte Verfahren bei Temperaturen oberhalb von $1000{ }^{\circ} \mathrm{C}$ [3]. Diese Verfahren sind jedoch hinsichtlich Flexibilität, Integrationsfähigkeit in elektronische Schaltungen und Miniaturisierbarkeit begrenzt [8].

Eine vielversprechende Alternative stellt das Verfahren der aerosolbasierten Kaltabscheidung (engl. Aerosol Deposition, AD) dar. Bei diesem neuartigen Beschichtungsverfahren können bei Raumtemperatur dichte keramische Schichten direkt aus einem keramischen Ausgangspulver auf unterschiedlichste Substratmaterialien (Glas, Keramik, Polymer, Me- 
tall) aufgebracht werden [9,10]. Allgemein zeichnen sich die aerosolkaltabgeschiedenen Schichten durch eine hervorragende Substratanbindung, eine hohe Dichtigkeit und Funktionseigenschaften aus, die mit denen der klassischen Volumenkeramiken vergleichbar sind. Ein schematischer Aufbau einer Anlage für die aerosolbasierte Kaltabscheidung ist in der nachfolgenden Abb. 1 dargestellt.

$x y$-Lineartisch mit

Substrathalter

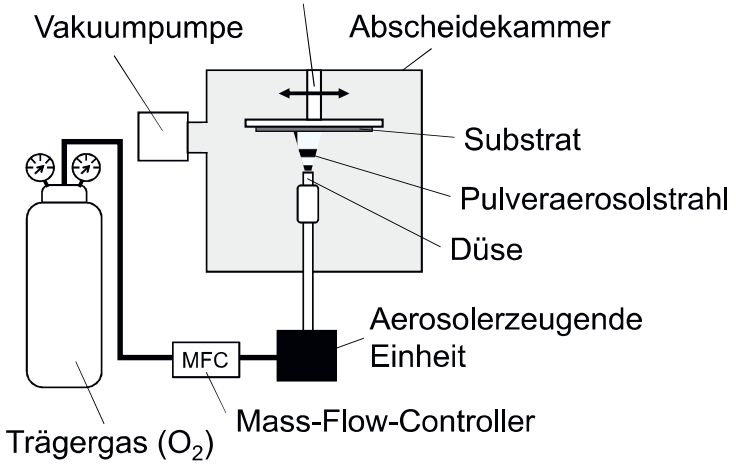

Abb. 1: Prinzipskizze einer Anlage für die aerosolbasierte Kaltabscheidung

Eine Anlage besteht grundsätzlich aus drei Hauptkomponenten - einer aerosolerzeugenden Einheit, einer Vakuumpumpe und einer Abscheidekammer. Während des Prozesses wird in der Abscheidekammer ein Vakuum erzeugt. In der aerosolerzeugenden Einheit wird das Ausgangspulver mit einem Trägergas (hier Sauerstoff) vermischt und ein Partikelaerosol erzeugt. Das generierte Partikelaerosol wird durch eine sich einstellende Druckdifferenz in die Abscheidekammer transportiert. Dabei passiert das Partikelaerosol eine Düse (hier Schlitzdüse $10 \mathrm{~mm} \times 0,5 \mathrm{~mm}$ ) und wird auf mehrere hundert Meter pro Sekunde beschleunigt. Die Partikel aus dem Aerosol treffen in der Abscheidekammer auf ein Substrat und bilden nach dem RTIC-Mechanismus (Room Temperature Impact Consolidation [10]) eine festanhaftende keramische Schicht aus.

Bisherige Untersuchungen an einem $\mathrm{NiMn}_{2} \mathrm{O}_{4}-$ Modellpulver konnten bereits zeigen, dass eine Abscheidung von dichten keramischen Schichten mit typischer NTCR-Charakteristik möglich ist [11-13]. Für eine industrielle Umsetzbarkeit des Verfahrens ist es jedoch erforderlich, dass auch industrienahe Pulver abgeschieden werden können. Dadurch könnte das Verfahren ohne große Änderungen in den Produktionsprozess integriert werden.

Im Folgenden wird daher untersucht, ob ein produktionsnahes Pulver mittels der aerosol- basierten Kaltabscheidung zu mechanisch stabilen Schichten mit typischer NTCRCharakteristik abgeschieden werden kann. Auch wird geklärt, ob eine gezielte Einstellung der Schichtdicke und damit des elektrischen Widerstandes möglich ist.

\section{Experimentelles}

Für die Untersuchung kam ein produktionsnahes Ni-Mn-Oxidpulver zum Einsatz. Um das Abscheideverhalten zu verbessern wurde das keramische Pulver durch Mahlen, Trocknen und Sieben AD-typisch präpariert. Das 30minütige Pulvermahlen erfolgte bei $400 \mathrm{~min}^{-1}$ mit Hilfe einer Kugelmühle (Pulverisette 5, Fritsch). Für die Mahlung kamen Mahltiegel und -kugeln ( $\varnothing 10 \mathrm{~mm}$ ) aus stabilisierten Zirkoniumdioxid und die Mahlflüssigkeit Cyclohexan (Sigma-Aldrich) zum Einsatz. Das Pulver wurde bei $200{ }^{\circ} \mathrm{C}$ in einem elektrisch betriebenen Umluftofen (UF110, Memmert) getrocknet. Für die Siebung wurde ein Sieb mit einer Maschenweite von $90 \mu \mathrm{m}$ eingesetzt.

Im Anschluss erfolgte die aerosolbasierte Kaltabscheidung des präparierten produktionsnahen Keramikpulvers auf Edelstahl- und $\mathrm{Al}_{2} \mathrm{O}_{3}$-Substraten. Um den Einfluss der Anzahl an Überfahrten des Substrathalters auf die Schichtdicke $z$ zu analysieren, wurde bei der aerosolbasierten Kaltabscheidung die Anzahl der Überfahrtsschleifen $N_{\text {schleife }}$ (zwei Überfahrten bilden eine Überfahrtsschleife) im Bereich von 1 bis 20 variiert. Die Charakterisierung der hergestellten Schichten fand hinsichtlich der mechanischen Schichtstabilität, der Schichtmorphologie und der Schichtdicke mittels Tape-Test, REM (Leo 1450 VP, Zeiss) und Tastschnittverfahren (Perthometer, PGK, S2, Mahr) statt.

Um zu untersuchen, ob die aerosolkaltabgeschiedenen Schichten die typische NTCRCharakteristik aufweisen und ob über eine Variation der Überfahrtsschleifen $N_{\text {schleife }}$ der elektrische Widerstand bei $25{ }^{\circ} \mathrm{C} \quad R_{25}$ gezielt eingestellt werden kann, wurden im zweiten Teil der Studie chipbasierte NTCR-Bauteile der Größe 1206 (1,6 mm x 3,2 mm) mit 10 und 20 Überfahrtsschleifen hergestellt. Die aerosolbasierte Kaltabscheidung des präparierten produktionsnahen Keramikpulvers erfolgte hierzu auf mit Sollbruchkanten und siebgedruckten Au-Interdigitalelektrodenstrukturen versehenen $\mathrm{Al}_{2} \mathrm{O}_{3}$-Substraten. Die Kontakte wurden während des Abscheidens mittels einer papierbasierten Einwegmaske abgedeckt. Nach dem Abscheiden fanden eine manuelle Vereinzelung der Bauteile sowie eine elektrische Charakterisierung im Temperaturbereich von $25^{\circ} \mathrm{C}$ 
bis $85^{\circ} \mathrm{C}$ statt. Die elektrische Messung erfolgte in einem Thermostatbad (SL-12, Julabo) mit einem niederviskosen Silikonöl (200 Fluid, 5 cst, Dow Corning) mittels Vierleitermesstechnik und unter Einsatz eines Digitalmultimeters (2700, Keithley). Aus den ermittelten $R-T$ Daten wurden anschließend die charakteristischen NTCR-Kenngrößen, der elektrische Widerstand bei $25^{\circ} \mathrm{C} R_{25}$ und die Thermistorkonstante $B(\mathrm{Gl}$. (1)) bestimmt.

\section{Ergebnisse}

Die Untersuchung konnte zeigen, dass die aerosolbasierte Kaltabscheidung eines produktionsnahen Keramikpulvers zu mechanisch stabilen Schichten möglich ist (keine Ablösung durch Tape-Test). Die Schichten zeichnen sich, wie in Abb. 2 ersichtlich, durch eine dichte, nanokristalline Schichtstruktur und eine hervorragende Substratanbindung aus.

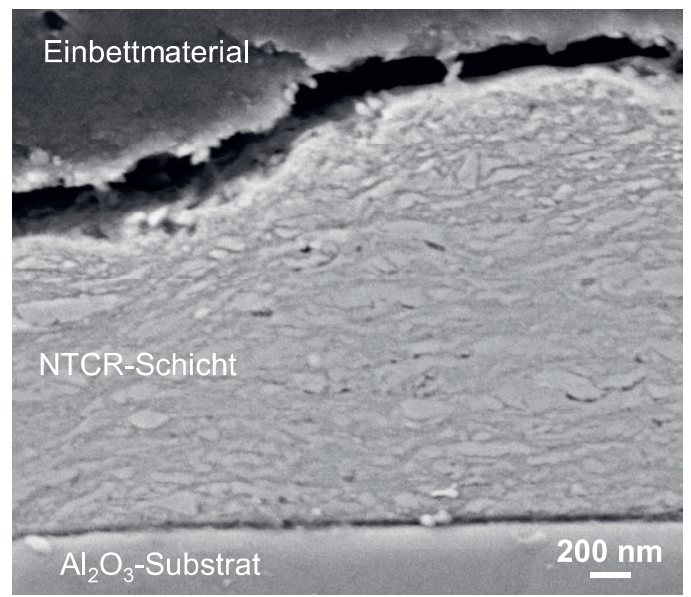

Abb. 2: Aerosolkaltabgeschiedene NTCRSchicht auf einem $\mathrm{Al}_{2} \mathrm{O}_{3}$-Substrat im Schliff

Auch konnte sowohl auf Edelstahl- als auch auf $\mathrm{Al}_{2} \mathrm{O}_{3}-\mathrm{Substraten}$ ein linearer Zusammenhang zwischen der Anzahl an Überfahrtsschleifen $N$ schleife und der Schichtdicke $z$ nachgewiesen werden (Abb. 3). Darüber hinaus konnte gezeigt werden, dass auf weicheren Substraten höhere Schichtdicken $z$ erzielt werden können. So liegen die Schichtdicken der aerosolkaltabgeschiedenen NTCR-Schichten beim Edelstahlsubstrat bei 10 Überfahrtsschleifen im Bereich von $25 \mu \mathrm{m}$ und bei 20 Überfahrtsschleifen sogar im Bereich von $50 \mu \mathrm{m}$. Im Gegensatz dazu beträgt die Schichtdicke der aerosolkaltabgeschiedenen NTCR-Schicht beim deutlich härteren $\mathrm{Al}_{2} \mathrm{O}_{3}$ Substrat bei 10 Überfahrtsschleifen nur etwa $2,5 \mu \mathrm{m}$ und bei 20 Überfahrtsschleifen nur etwa $6 \mu \mathrm{m}$.

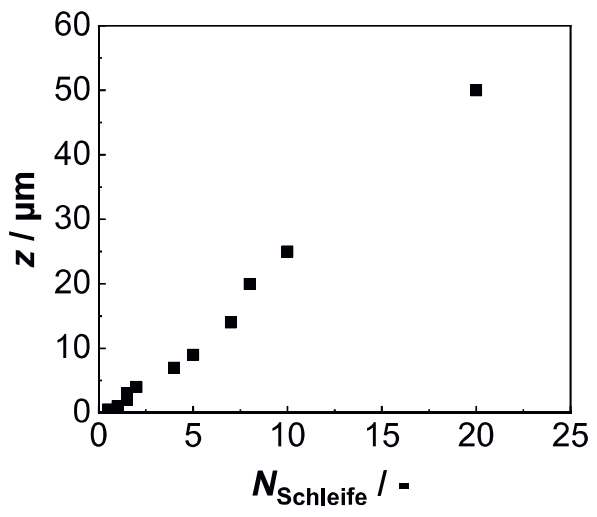

Abb. 3: Schichtdicke $z$ in Abhängigkeit der Anzahl an Überfahrtsschleifen $N$ schleife auf Edelstahlsubstraten

Basierend auf diesen Erkenntnissen, wurden im zweiten Teil der Arbeit chipbasierte NTCRBauteile mit 10 und 20 Überfahrtsschleifen hergestellt. Hierbei konnte nachgewiesen werden, dass mittels der aerosolbasierten Kaltabscheidung des produktionsnahen Keramikpulvers erfolgreich Chipbauteile der Baugröße 1206 hergestellt werden können. Wie in Abb. 4 zu erkennen, sind die abgeschiedenen NTCRSchichten auch nach der Demaskierung und der manuellen Vereinzelung der Bauteile stabil und zeigen keinerlei Risse oder Abplatzungen. Auch liegen die Kontaktpads vollständig unbeschichtet vor, was eine erfolgreiche Maskierung während der aerosolbasierten Kaltabscheidung bestätigt. Die elektrische Charakterisierung der Bauteile zeigt, dass diese reproduzierbar das typische NTCR-Verhalten eines annähernd exponentiellen Abfalls des elektrischen Widerstandes mit steigender Temperatur aufweisen.

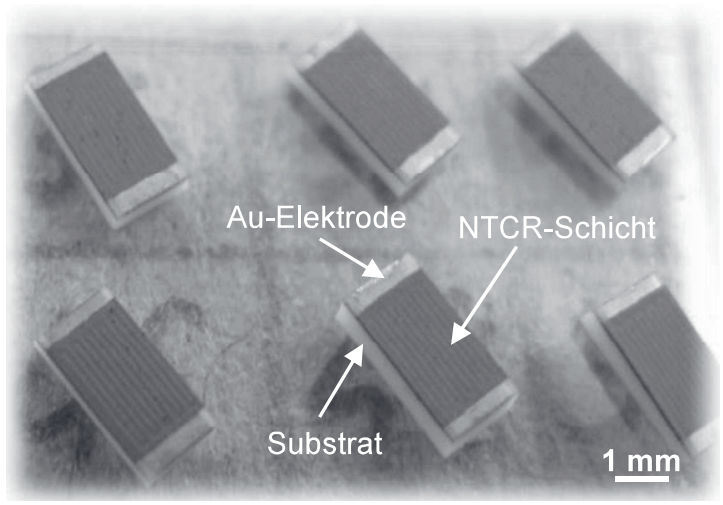

Abb. 4: Chipbasierte NTCR-Bauteile der Baugröße 1206 nach der manuellen Vereinzelung

Ein Vergleich der NTCR-Kenngrößen $R_{25}$ und $B$ von Bauteilen, die mit 10 und 20 Überfahrtsschleifen $N_{\text {schleife }}$ hergestellt wurden, ist in Abb. 
5 dargestellt. Es zeigt sich, dass der $B$-Wert in Abb. $5 \mathrm{a}$ ) erwartungsgemäß unabhängig von der Anzahl an Überfahrtsschleifen Nschleife ist. Mit $3865 \mathrm{~K}$ liegt der $B$-Wert zudem reproduzierbar im Bereich derer von konventionell gesinterten Bauteilen. Im Gegensatz dazu zeigt sich beim $R_{25}$ in Abb. 5 b) ein deutlicher Einfluss der Anzahl an Überfahrtsschleifen $N$ schleife. So verringert sich der $R_{25}$ reproduzierbar von etwa $35 \mathrm{k} \Omega$ bei 10 Überfahrtsschleifen auf $14 \mathrm{k} \Omega$ bei 20 Überfahrtsschleifen. Die Abnahme des elektrischen Widerstandes $R$ mit einer steigenden Anzahl an Überfahrtsschleifen ist gemäß der Beziehung $R \sim 1 / z$ auf eine Zunahme der Schichtdicke $z$ der aerosolkaltabgeschiedenen NTCR-Schicht zurückzuführen. So weisen die Bauteile, die auf Basis von 10 Überfahrtsschleifen hergestellt wurden, eine NTCR-Schichtdicke im Bereich von 2,5 $\mu \mathrm{m}$ und die Bauteile auf Basis von 20 Überfahrtsschleifen eine NTCR-Schichtdicke im Bereich von $6 \mu \mathrm{m}$ auf.
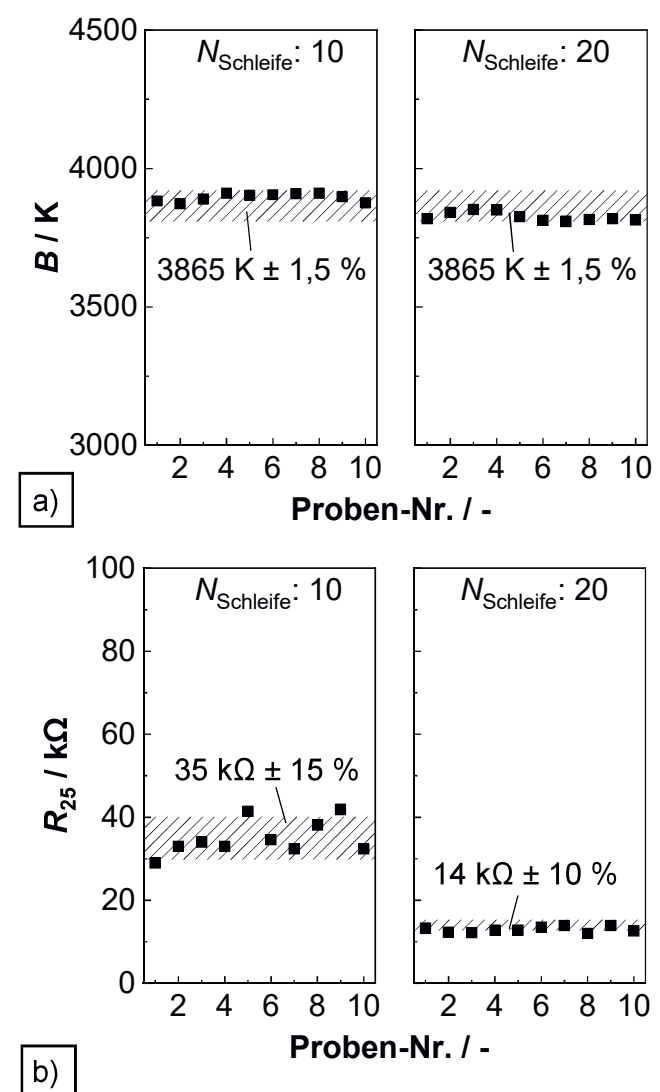

Abb. 5: a) Ermittelter $B$-Werte und b) $R_{25}$ Werte von chipbasierten Bauteilen in Abhängigkeit der Anzahl an Überfahrtsschleifen $N_{\text {schleife }}$

Hervorzuheben ist hierbei, dass die erzielten Schichtdicken der aerosolkaltabgeschiedenen Schicht sehr gut mit den Schichtdicken der aerosolkaltabgeschiedenen NTCR-Schichten auf den $\mathrm{Al}_{2} \mathrm{O}_{3}$-Substraten aus Versuchsteil eins übereinstimmen. Folglich ist es möglich durch eine Variation der Anzahl an Überfahrtsschleifen gezielt die Schichtdicke der aerosolkaltabgeschiedenen NTCR-Schicht und damit deren Widerstand einzustellen.

\section{Zusammenfassung}

In der vorliegenden Arbeit konnte nachgewiesen werden, dass eine aerosolbasierte Kaltabscheidung eines produktionsnahen Keramikpulvers zu mechanisch stabilen Schichten mit typischer NTCR-Charakteristik möglich ist. Sowohl auf unstrukturierten Edelstahl- und $\mathrm{Al}_{2} \mathrm{O}_{3}$-Substraten als auch auf strukturierten $\mathrm{Al}_{2} \mathrm{O}_{3}$-Substraten konnte die Schichtdicke der aerosolkaltabgeschiedenen Schicht und damit der elektrische Widerstand gezielt über die Anzahl an Überfahrtsschleifen variiert und damit eingestellt werden.

\section{Literaturnachweis}

[1] M. Parlak, T. Hashemi, M. J. Hogan, A. W. Brinkman, Thin Solid Films 345, 307-311 (1999); doi: 10.1016/S0040-6090(98)01426-6

[2] K. Park, D. Y. Bang, J. Mater. Sci.: Mater. Electron. 14, 81-87 (2003); doi: 10.1023/A:1021900618988

[3] A. Feteira, J. Am. Ceram. Soc. 92, 967-983 (2009); doi: 10.1111/j.1551-2916.2009.02990.x

[4] G. D. C. Csete de Györgyfalva, I. M. Reaney, J. Eur. Ceram. Soc., 21, 2145-2148 (2001), doi: 10.1016/S0955-2219(01)00190-X

[5] R. Schmidt, A. Basu, A. W. Brinkman, Phys. Rev. B, 72, 115101 (2005), doi: 10.1103/PhysRevB.72.115101

[6] P. Fau, J. P. Bonino, J. J. Demai, A. Rousset, Appl. Surf. Sci. 65/66, 319-324 (1993); doi: 10.1016/0169-4332(93)90679-6

[7] A. Rousset, R. Legros, A. Lagrange, J. Eur. Ceram. Soc. 13, 185-195 (1994); doi: 10.1016/0955-2219(94)90027-2

[8] A. Darmawansyah, Prosiding SENTIA 2009, 12.03.2009, Malang, Indonesia

[9] D. Hanft, J. Exner, M. Schubert, T Stöcker, P. Fuierer, R. Moos, J. Ceram. Sci. Technol. 2015, 6, 147-182; doi: 10.4416/JCST2015-00018

[10] J. Akedo, J. Therm. Spray Technol. 2008, 17, 181-198; doi: 10.1007/s11666-008-9163-7

[11] M. Schubert, C. Münch, S. Schuurman, V. Poulain, J. Kita, R. Moo, J. Eur. Ceram. Soc. 38, 613-619 (2018); doi: 10.1016/j.jeurceramsoc.2017.09.005

[12] M. Schubert, C. Münch, S. Schuurman, V. Poulain, J. Kita, R. Moos, Sensors 18, 3982 (2018); doi: $10.3390 / \mathrm{s} 18113982$

[13] M. Schubert, J. Kita, C. Münch, R. Moos, Funct. Mater. Lett. 10, 1750073 (2017); doi: 10.1142/S1793604717500734 